\title{
Optimal control of a quasi-variational obstacle problem
}

\author{
Samir Adly • Matine Bergounioux • \\ Mohamed Ait Mansour
}

Received: date / Accepted: date

\begin{abstract}
We consider an optimal control where the state-control relation is given by a quasi-variational inequality, namely a generalized obstacle problem. We give an existence result for solutions to such a problem. The main tool is a stability result, based on the Mosco-convergence theory, that gives the weak closeness of the control-to-state operator. We end the paper with some examples.
\end{abstract}

Keywords Optimal control, quasi-variational inequalities, Mosco convergence.

\section{Introduction}

Optimal control of problems governed by PDE's have been extensively studied for many years. Then people investigated problems governed by variational inequalities (see [5] for example) from many points of view. Next challenge is the optimal control of problems whose state "equation" is a quasi-variational inequality (QVI). A first step has been done, considering problems where the control function is part of the variational inequality [7]. Now we are interested in the following

$$
(\mathcal{P}) \quad \min \left\{J(y, f), y \in \mathcal{T}(f), f \in U_{a d} \subset U\right\},
$$

where $\mathcal{T}$ is a set-valued operator which associates to $f$, the set of elements $y$ solution(s) to

$$
\forall z \in K(y, f), \quad\langle\mathcal{A}(y, f), z-y\rangle \geq 0 ;
$$

The research of S. Adly has been supported by the "Fondation EADS" and the ANR project "Guidage".

S. Adly,

XLIM UMR-CNRS 6172, Université de Limoges, 87060 Limoges, France

E-mail: samir.adly@unilim.fr

M. Bergounioux

UMR 6628-MAPMO, Fédération Denis Poisson, Université d'Orléans, BP 6759, F-45067

Orléans Cedex 2,

E-mail: maitine.bergounioux@univ-orleans.fr

M. Ait Mansour,

Facult Poly-Disciplinaire, route Sidi Bouzid BP 4162 - 46000 Safi, Morocco.

E-mail: maitmansour@hotmail.com 
here $K$ is a set-valued application from $X \times U$ to $2^{X}, X$ and $U$ are Banach and Hilbert spaces respectively. Let us give an example: let $Y$ be a Banach space and $A$ a differential operator (linear or not), parabolic or elliptic from $Y$ to the dual space $Y^{\prime}$, and $\Xi$ an application from $\mathbb{R} \times \mathbb{R} \times \mathbb{R}$ to $\mathbb{R}$. We identify $\Xi$ and the associated Nemitsky operator. The differential equation that relates the control $f$ to the state function $y$ (i.e., the state "equation") is

$$
\langle A y, z-y\rangle_{Y, Y^{\prime}}+\Xi(y, z, f)-\Xi(z, z, f) \geq(f, z-y) \quad \forall z \in Y,
$$

where, for example

1. $\Xi(y, z, f)=\Xi(z)$ gives the classical variational inequalities;

2. $\Xi(y, z, f)=\Xi(f, z)$ gives (for example) obstacle problems (where the obstacle is the control) as in [7];

The full dependence of $\Xi$ with respect to $(y, z, f)$ leads to quasi-variational inequalities: this is the problem we are interested in.

To get existence results for solutions to problem $(\mathcal{P})$ we need continuity/stability properties for the state-control operator $\mathcal{T}$. So, we have to study precisely the quasivariational inequalities from this point of view.

Let us mention that few people has been investigating optimal control problems for quasi-variational inequalities. H. Dietrich [10] has been considering problems where $\Xi(y, z, f)$ is the value at $z$ of the indicatrix function of a set $K(y)=g(y)+C$ where $C$ is constant and $g$ is a $\mathcal{C}^{1}$ function, using a smooth dual gap function [9]. In our paper, we adopt an abstract point of view and give generic assumptions to get existence in a general context.

The paper is organized as follows. We first present the problem and recall classical tools and definitions. In Section 3, we give an existence result for solution to the quasivariational inequality. Next Section is devoted to stability results that allow to give a weak closeness property of the the state-control operator $\mathcal{T}$. In last section we prove that the optimal control problem has at least one optimal solution and give many examples.

\section{The basic quasi-variational inequality problem}

In this section we present the QVI and recall some classical definitions. Then we give an existence result for this QVI.

\subsection{Setting the QVI problem}

Let us recall what a quasi-variational inequality is in an abstract setting. Given a closed convex set $D$ of a vector topological space $X$, a real-valued function $\varphi: D \times D \rightarrow \mathbb{R}$ and an extended real-valued function $\Sigma: D \times D \rightarrow \mathbb{R} \cup\{+\infty\}$, we introduce the following abstract quasi-variational inequality: find $\bar{x} \in D$ such that

$$
\varphi(\bar{x}, y)+\Sigma(\bar{x}, y)-\Sigma(\bar{x}, \bar{x}) \geq 0 \quad \forall y \in D .
$$

In the present paper, having in mind some applications, we focus on the so-called "obstacle problem" : given $K: D \rightrightarrows X$, find $\bar{y} \in K(\bar{y})$ such that

$$
\varphi(\bar{x}, y)+\Phi(y)-\Phi(\bar{x}) \geq 0, \quad \forall y \in K(\bar{x}),
$$


where $K: D \rightrightarrows X$, is a multivalued application from $D$ to $2^{X}$ (this is the meaning of the notation " $D \rightrightarrows X$ ") and $\Phi$ is an extended real-valued function from $D$ to $\mathbb{R} \cup\{+\infty\}$; here we have set $\Sigma(\bar{x}, y)=1_{K(\bar{x})}+\Phi(y)$, where $1_{C}$ denotes the indicatrix function of the set $C$ :

$$
1_{C}(x)= \begin{cases}0 & \text { if } x \in C, \\ +\infty & \text { else }\end{cases}
$$

Problem (2.1) was considered earlier by Mosco and Joly [17] in regard to existence theory in the coercive setting, and recently studied by Bazán [13] again from the point of view existence in the noncoercive framework. Problem (2.1) covers other problems more than those quoted in these previous works: it still therefore deserves a further treatment. We have to notice that the function $\Sigma$ in (2.1) has been introduced to reflect the dependence with respect to the constraints on the solutions while the term $\Phi(y)-\Phi(\bar{x})$ can not be contained in $\varphi$ since $\Phi$ may takes the infinity as a value. As confirmed by the existing literature, from the stability point of view, only few efforts have been dedicated to quasi-variational inequalities. Some qualitative results were established by Morgan and Lignola in [21] for the case $\Phi=0$ and the obtained properties can be regarded as a closeness of the solution map, which is intimately related to upper approximation of solutions.

In [20] QVI solutions existence was considered via Tychonov well-posedness tool. The paper is henceforth devoted to the well-posedness properties of QVI. This leads to existence/uniqueness results but assumptions and techniques are quite different from ours. From another point of view, in [3] the authors focus on the (differential) set-valued operator (say $T$ ) defining the QVI. They investigate quasi-monotonicity properties that we do not consider in this paper. We assume (in as standard way) that the operator $(A)$ is single-valued and monotone.

Note that results of [1] cannot be applied here since the framework is completely different. We deal with a general constraint set-valued operator $K$ and look for existence results (via stability) in infinite dimensional spaces, that is not the case in [1] .

Throughout this paper $V$ will be a reflexive Banach space whose topological dual, duality pairing and norm are denoted by $V^{\prime},\langle.,$.$\rangle and \|$.$\| respectively. The norm of$ $V^{\prime}$ will be denoted by $\|.\|_{*}$. The symbol $\rightarrow$ (resp. $\rightarrow$ ) will stand for the strong (resp. weak) convergence. The control function (that is fixed in a first step ) $u$ belongs to an Hilbert space $U$. Let us give

- operators $A: V \rightarrow V^{\prime}$ and $B: U \rightarrow V^{\prime}$

- a set-valued map $K: V \rightrightarrows V$ with nonempty closed convex values. Note that $K$ may involve a "constant" part that may represent classical state constraints. For example, $K(y)=\tilde{K}(y) \cap C$ where $\tilde{K}: V \rightrightarrows V$ and $C$ is a non empty, convex subset of $V$.

- a convex extended real-valued function $\Phi: V \times U \rightarrow \mathbb{R} \cup\{+\infty\}$, whose properties will be made precise in the sequel. Note that the convex $C$ mentioned above can be the domain of $\Phi$.

The corresponding quasi-variational inequality problem, $\mathrm{QVI}(A, u, \Phi, K)$, is to find $\bar{y} \in K(\bar{y})$ such that

$$
\langle A \bar{y}-B u, y-\bar{y}\rangle+\Phi(y, u)-\Phi(\bar{y}, u) \geq 0, \quad \forall y \in K(\bar{y}),
$$

Many problems arising in optimization, economic equilibrium [14], calculus of variations, free boundary problems [4], feasibility in optimal control as well as in mechanic 
[12] can be modelled by (2.3). We shall assume that

$$
\forall x \in V \quad 0 \in K(x)
$$

This assumption is consistent with the applications we present at the end of this paper. We recall now some variational analysis basic concepts, that we need in the sequel.

\subsection{Hemicontinuity, semicontinuity and monotonicity}

Let $X$ and $Y$ be two Hausdorff topological spaces and let $\Gamma: X \rightrightarrows Y$ be a set-valued map. Recall that the domain of $\Gamma$ is $\operatorname{Dom}(\Gamma)=\{\mathrm{x} \in \mathrm{X} \mid \Gamma(\mathrm{x}) \neq \emptyset\}$. Its graph is the set

$$
\operatorname{graph}(\Gamma)=\{(\mathrm{x}, \mathrm{y}) \in \mathrm{X} \times \mathrm{Y} \mid \mathrm{x} \in \operatorname{Dom}(\Gamma), \mathrm{y} \in \Gamma(\mathrm{x})\}
$$

- If graph $(\Gamma)$ is closed (resp. convex), we say that $\Gamma$ is closed (resp. convex).

$-\Gamma$ is upper (resp. lower) semicontinuous at $\bar{x} \in X$ if for any open $U$ in $Y$ with $\Gamma(\bar{x}) \subset U$ (resp. $\Gamma(\bar{x}) \cap U \neq \emptyset)$ the set $\{x \in X \mid \Gamma(x) \subset U\}$ (resp. $\{x \in$ $X \mid \Gamma(x) \cap U \neq \emptyset\})$ is open in $X$.

$-\Gamma$ is said to be continuous at $\bar{x}$ if it is both upper and lower semicontinuous at $\bar{x}$.

Let us mention that if $\Gamma$ is upper semicontinuous, then it is closed. If in addition, the range of $\Gamma$ is compact, then $\Gamma$ is upper semicontinuous if and only if $\Gamma$ is closed. For a discussion on this topic and related continuity properties we refer for example to [2], [8] and references cited therein.

A single valued operator $A: X \rightarrow X^{\prime}$ is said to be hemicontinuous [5] if, for all $u, v \in X$,

$$
w-\lim _{\lambda \rightarrow 0} A(u+\lambda v)=A u .
$$

An operator $A: X \rightarrow X^{\prime}$ is said to be monotone if

$$
\langle A(u)-A(v), u-v\rangle \geq 0 \quad \forall u, v \in X
$$

and $M$-strongly monotone if there is some $M>0$ such that

$$
\langle A(u)-A(v), u-v\rangle \geq M\|u-v\|^{2} \quad \forall u, v \in X .
$$

Most of existence schemes of solutions to quasi-variational inequalities use the recurrent tool : define a suitable set-valued map related to the data of the problem and look for its fixed points. There are many results of fixed points of set-valued maps e.g. the Kakutani-Ky Fan's result which is an extension of the topological Brouwer's fixed point: any self upper semicontinuous set-valued map with nonempty compact and convex values has a fixed point, (see $[4, ?]$ for example).

\subsection{Mosco convergence}

Let $\left(K_{n}\right)_{n}$ be a sequence of subsets of $V$. We recall basic definitions on set convergence:

Definition 1 Let $\left(K_{n}\right)_{n}$ be a sequence of nonempty closed convex subsets of $V$. We say that $K_{n}$ converges to $K$ (a closed closed convex subset of $V$ ) in the sense of Mosco if the following two assumptions are satisfied 
(i) If $\left(v_{n}\right)_{n}$ weakly converges to $v$ with $v_{n} \in K_{n}$ for $n$ large enough, then the weak limit $v \in K$

(ii) For any $v \in K$, there exists a sequence $\left(v_{n}\right)_{n}$ strongly converging to $v$ such that $v_{n} \in K_{n}$ for every $n$ large enough.

Remark 1 The above assumptions (i) and (ii) in Definition 1 can be summarized as

$$
w-\limsup _{n} K_{n} \subseteq K \text { and } s-\liminf _{n} K_{n} \supseteq K,
$$

where $s-\liminf$ and $w-\lim \sup$ denotes the inferior strong limit and the superior weak limit respectively in the sense of Kuratowski-Painlevé. We note also that, since strong convergence implies weak convergence, (i) and (ii) are equivalent to

$$
w-\limsup _{n} K_{n} \subseteq K \subseteq w-\liminf _{n} K_{n} \text { and } s-\limsup _{n} K_{n} \subseteq K \subseteq s-\liminf _{n} K_{n} .
$$

We can find some further characterizations of the Mosco-convergence by using projections, distance functions and the convergence of Yosida approximations in [11], [26], $[24]$.

In the very case where the sequence $K_{n}$ is related to a set-valued application we may formulate the Mosco-convergence as follows :

Definition 2 Let $K: V \rightrightarrows V$ a set-valued application. For any $v \in V$ and any sequence $\left(v_{n}\right)_{n \in \mathbb{N}}$ (weakly) converging to $\mathrm{v}$, we say that the sequence of sets $K\left(v_{n}\right)$ Mosco-converges to $K(v)$ if and only if :

(i) For every sequence $y_{n} \in K\left(v_{n}\right)$ such that $y_{n}$ weakly converges to $y$, then $y \in K(v)$. (ii) For every $y \in K(v)$, there exists $y_{n} \in K\left(v_{n}\right)$ (for $n$ large enough) such that $y_{n}$ strongly converges to $y$.

In Section 5. we give an example of Mosco-convergence related to the control problem we study in the sequel.

\section{Existence of solutions to QIV Problem (2.3)}

In what follows the control function $u$ is fixed and we set $f=B u \in V^{\prime}$ in the sequel. For the sake of simplicity, we do not indicate the dependence of $\Phi$ with respect to $u$ and denote $\Phi(., u):=\Phi_{u}$ for the fixed value of the control parameter $u \in U$.

Let us introduce the map $\mathbb{S}_{u}: V \rightrightarrows V$ defined by

$$
\mathbb{S}_{u}(x)=\left\{y \in V \mid\langle A y-f, z-y\rangle+\Phi_{u}(z)-\Phi_{u}(y) \geq 0, \forall z \in K(x)\right\},
$$

or equivalently

$$
\mathbb{S}_{u}(x)=\bigcap_{z \in K(x)} F_{x}(z)
$$

where $F_{x}(z)=\left\{y \in V \mid\langle A y-f, z-y\rangle+\Phi_{u}(z)-\Phi_{u}(y) \geq 0\right\}$.

Clearly, the solutions of problem (2.3) are fixed points of the map $\mathbb{S}_{u}$. Therefore, the scheme of existence of solutions to this problem is based on the two following essential steps:

- $\mathbb{S}_{u}$ is nonempty-valued i.e., $\bigcap_{z \in K(x)} F_{x}(z) \neq \emptyset$ for every $x$; 
$-\mathbb{S}_{u}$ admits at least a fixed point.

When it is nonempty, the set of fixed points of $\mathbb{S}_{u}$ will be denoted by $\mathbf{F P}\left(\mathbb{S}_{u}\right)$. The operator $A$, a closed convex subset $D$ of $V$ and $f \in V^{\prime}$ being given, we call $\mathcal{S}_{u}(D)$ the solutions set to the following variational inequality $\operatorname{VI}(D)$ : find $y \in D$ such that

$$
\langle A y-f, z-y\rangle+\Phi_{u}(z)-\Phi_{u}(y) \geq 0, \forall z \in D .
$$

We look for the fixed points of $\mathbb{S}_{u}$ where $\mathbb{S}_{u}(x)=\mathcal{S}_{u}(K(x))$. If monotonicity and convexity assumptions occur, a classical tool is to consider the Minty's variational inequality : find $y \in D$ such that

$$
\langle A z-f, z-y\rangle+\Phi_{u}(z)-\Phi_{u}(y) \geq 0, \forall z \in D .
$$

Let us call $\mathcal{S}_{u}^{M}(D)$ the solutions set of (3.3).

Lemma 1 If $\Phi_{u}$ is proper, convex and lower-semicontinuous, then for every $x \in V$, $\mathcal{S}_{u}^{M}(K(x))$ is closed and convex, possibly empty. In addition, if $A$ is monotone, hemicontinuous then

(i) $\mathcal{S}_{u}(K(x))=\mathcal{S}_{u}^{M}(K(x))$.

(ii) $\mathbb{S}_{u}$ is closed and convex valued on its domain.

Proof.- The first point is a classical result ( see [4,?] for example) . Point (ii) is a direct consequence of point (i).

We turn now our attention to existence results for problem (2.3) when the operator $A$ is strongly monotone and hemicontinuous and $u \in U$ is fixed.

Theorem 1 Let be $u \in U$ Assume the following holds:

(i) $A: V \rightarrow V^{\prime}$ is hemicontinuous and $M$-strongly monotone;

(ii) $A$ is bounded and

$$
\forall y_{n} \rightarrow y, \forall z_{n} \rightarrow z \quad\langle A(y), z-y\rangle \leq \liminf _{n}\left\langle A\left(y_{n}\right), z_{n}-y_{n}\right\rangle .
$$

(iii) For all $\left(x_{n}\right)_{n}$ in $V$ such that $x_{n} \rightarrow x$, then $K\left(x_{n}\right)$ Mosco-converges to $K(x)$;

(iv) We assume that $\Phi_{u}: V \rightarrow \mathbb{R}$ is convex and continuous and either

(a) $\Phi_{u}$ is L-Lipschitz continuous with $L>0$

or

(b) $\Phi_{u}$ satisfies

$$
\min _{v \in V} \Phi_{u}(v)=\Phi_{u}(0)
$$

Then problem (2.3) admits at least one solution.

Proof.- - We first prove that $\mathbb{S}_{u}$ is (graph) weakly- closed, that is :

$$
\text { if }\left(x_{n}, y_{n}\right) \rightarrow(x, y) \text { with } y_{n} \in \mathbb{S}_{u}\left(x_{n}\right) \text { then } y \in \mathbb{S}_{u}(x) \text {. }
$$

Let $\left(x_{n}\right)_{n \in \mathbb{N}}$ be a sequence weakly convergent to $x \in V$. Thanks to assumptions (i) and (iv) we know that

$$
\forall n \quad \mathcal{S}_{u}\left(K\left(x_{n}\right)\right) \neq \emptyset,
$$

(one can refer to [5] for example). The strong monotonicity of $A$ ensures the uniqueness of the solution to $\mathrm{VI}\left(A, f, \Phi_{u}, K\left(x_{n}\right)\right)$ for every $n$ so that

$$
\mathbb{S}_{u}\left(x_{n}\right)=\left\{y_{n}\right\}
$$


We assume that $y_{n} \rightarrow y$; we have to prove that $y \in \mathbb{S}_{u}(x)$. Let $z$ be an arbitrary point of $K(x)$. From (iii) there exists $z_{n} \in K\left(x_{n}\right)$ such that $z_{n} \rightarrow z$ strongly in $V$. This implies that $\lim _{n \rightarrow+\infty} \Phi_{u}\left(z_{n}\right)=\Phi_{u}(z)$. As $y_{n} \in \mathcal{S}_{u}\left(K\left(x_{n}\right)\right)$ we get

$$
\left\langle A y_{n}-f, z_{n}-y_{n}\right\rangle+\Phi_{u}\left(z_{n}\right)-\Phi_{u}\left(y_{n}\right) \geq 0
$$

In addition $y_{n} \in \mathcal{S}_{u}^{M}\left(K\left(x_{n}\right)\right)$ (cf. Lemma 1) so that

$$
\left\langle A z_{n}-f, y_{n}-z_{n}\right\rangle+\Phi_{u}\left(y_{n}\right)-\Phi_{u}\left(z_{n}\right) \leq 0 .
$$

Moreover $\Phi_{u}$ is continuous and convex, so it is weakly lower semi-continuous so that $\Phi_{u}(y) \leq \liminf _{n} \Phi_{u}\left(y_{n}\right)$. Using (ii) this yields that

$$
\langle A z-f, y-z\rangle+\Phi_{u}(y)-\Phi_{u}(z) \leq \liminf _{n}\left\langle A z_{n}-f, y_{n}-z_{n}\right\rangle+\liminf _{n}\left(\Phi_{u}\left(y_{n}\right)-\lim _{n} \Phi_{u}\left(z_{n}\right)\right) \leq 0 \text {. }
$$

So

$$
\langle A z-f, z-y\rangle+\Phi_{u}(z)-\Phi_{u}(y) \geq 0, \quad \forall z \in K(x) .
$$

This means that $y \in \mathcal{S}_{u}^{M}(K(x))=\mathcal{S}_{u}(K(x))=\mathbb{S}_{u}(x)$.

- We prove now that $\mathbb{S}_{u}$ has at least a fixed point. We have shown at the beginning of the proof that

$$
\forall x \in V \quad \mathcal{S}_{u}(K(x))=\mathbb{S}_{u}(x)=\left\{y_{x}\right\} .
$$

Therefore $\mathbb{S}_{u}$ is single-valued. Moreover, $0 \in K(x)$ (assumption (2.4)) implies that

$$
\left\langle A y_{x}-f,-y_{x}\right\rangle+\Phi_{u}(0)-\Phi_{u}\left(y_{x}\right) \geq 0 .
$$

Therefore, with the strong monotonicity of $A$, we obtain

$$
M\left\|y_{x}\right\|^{2} \leq\left\langle A y_{x}-A(0), y_{x}\right\rangle \leq \Phi_{u}(0)-\Phi_{u}\left(y_{x}\right)+\left\langle f-A(0), y_{x}\right\rangle .
$$

If assumption (iv)-(a) is satisfied, then using (3.5), we get

$$
M\left\|y_{x}\right\|^{2} \leq L\left\|y_{x}\right\|+\left\langle f-A(0), y_{x}\right\rangle .
$$

Thus, $\left\|y_{x}\right\| \leq c\|f\|_{*}$ with $c=c_{f, L, M}=\frac{\|f-A(0)\|_{*}+L}{M}(c$ is independent of $x)$.

If assumption (iv)-(b) is satisfied, then using (3.5), we have

$$
M\left\|y_{x}\right\|^{2} \leq\left\langle f-A(0), y_{x}\right\rangle \text {. }
$$

Thus, $\left\|y_{x}\right\| \leq c\|f\|_{*}$ with $c=c_{f, M}=\frac{\|f-A(0)\|_{*}}{M}(c$ is independent of $x)$.

Consider the convex weakly compact $C_{0}:=\bar{B}(0, c)$ of $V$ : we observe that $\mathbb{S}_{u}\left(C_{0}\right) \subset C_{0}$. As $\mathbb{S}_{u}$ is single valued and weakly closed we may use Schauder-Tychonoff theorem (see e.g. [15] page 147 Theorem 1.10 ) to ensure the existence of a fixed point of $\mathbb{S}$ in $C_{0}$.

Remark 2 The above result is a general existence result. In particular the assumption (iii) is a strong assumption which involves compactness of $K$. Many other existence results can be found in the literature without such hypothesis but only for some specific situations; as often the underlying compactness assumption is replaced by monotonicity assumptions. We refer to [6] for more details. 


\section{Stability results for problem (2.3) with respect to $f$ perturbations}

Although the generality is of great interest, we consider now the classical functional framework for PDE's control.

Let $V, H$ be Hilbert spaces such that $V \subset H$ with continuous, compact and dense embedding. $V^{\prime}$ denotes the dual of $V$. The control space $U$ is an Hilbert space as well and $U_{a d}$ is the set of admissible control functions : it a non empty, convex closed subset of $U$. The quasi-variational problems: $\mathrm{QVI}(\mathrm{A}, \mathrm{u}, \Phi, \mathrm{K})$ turns to be:

$$
\text { find } y_{u} \in K\left(y_{u}\right):\left\langle A y_{u}-B u, y-y_{u}\right\rangle+\Phi(y, u)-\Phi\left(y_{u}, u\right) \geq 0, \forall y \in K\left(y_{u}\right) \text {. }
$$

In the previous section, we proved that for every $u \in U$ the set of solutions to $\mathrm{QVI}(\mathrm{A}, \mathrm{u}, \Phi, \mathrm{K})$ is non empty (under assumptions of Theorem 1). So we may define the solution map of problem (4.1) that we denote by

$$
\mathcal{T}: u \mapsto \mathcal{T}(u)
$$

which is set-valued. We now establish the weak sequential closeness of $\mathcal{T}$.

Theorem 2 Let be $u \in U$ and $\left(u_{n}\right)_{n \in \mathbb{N}} \in U$ a sequence weakly convergent to $u$ in $U$. Assume the following:

(i) The set-valued map $K$ is closed and convex valued, i.e. $K(w)$ is closed and convex for all $w \in V$;

(ii) $A: V \rightarrow V^{\prime}$ is hemicontinuous, strongly monotone and bounded;

(iii) for every sequence $\left(y_{n}\right)_{n \in \mathbb{N}}$ strongly convergent to $y$ in $V$ and $\left(z_{n}\right)_{n \in \mathbb{N}}$ weakly convergent to $z$ in $V$

$$
\langle A(y), z-y\rangle \leq \liminf _{n}\left\langle A\left(y_{n}\right), z_{n}-y_{n}\right\rangle
$$

(iv) $B: U \rightarrow V^{\prime}$ is a compact operator;

(v) For every $x_{n}$ such that $x_{n}$ weakly converges to $x$ in $V$, then $K\left(x_{n}\right)$ Mosco-converges to $K(x)$.

(vi) $\Phi: V \times U \rightarrow \mathbb{R}$, is continuous and convex with respect to $y \in V$ and either,

- $\Phi$ is L-Lipschitz continuous with respect to $y \in V$ uniformly with respect to u i.e.

$$
\forall u \in U \quad|\Phi(y, u)-\Phi(z, u)| \leq L\|y-z\|,
$$

where $L>0$ is independent on $u$,

or

$$
\forall u \in U \quad \min _{y \in V} \Phi(y, u)=\Phi(0, u) .
$$

Moreover it must satisfies

(a) For every $x_{n} \in \mathcal{T}\left(u_{n}\right)$ such that: $x_{n} \rightarrow x$ (weakly in $V$ )

$$
\Phi(x, u) \leq \liminf _{n} \Phi\left(x_{n}, u_{n}\right) .
$$

(b) For every $x_{n} \in \mathcal{T}\left(u_{n}\right)$ such that: $x_{n} \rightarrow x$ (strongly in $V$ ), there are subsequences $\left(x_{n_{k}}\right)$ and $\left(u_{n_{k}}\right)$ of $\left(x_{n}\right)$ and $\left(u_{n}\right)$ respectively such that

$$
\underset{k}{\limsup } \Phi\left(x_{n_{k}}, u_{n_{k}}\right) \leq \Phi(x, u) .
$$


Then

1. There exists a constant $\kappa_{u}$ depending on $u$ such that

$$
\bigcup_{n \in \mathbb{N}} \mathcal{T}\left(u_{n}\right) \subset \mathcal{B}\left(0, \kappa_{u}\right),
$$

where $\mathcal{B}\left(0, \kappa_{u}\right)$ is the $V$-ball of radius $\kappa_{u}$.

2. For every $y_{n} \in \mathcal{T}\left(u_{n}\right)$, the sequence $y_{n}$ weakly converges (up to a subsequence) to some $y \in \mathcal{T}(u)$.

Remark 3 Note that

- assumptions (i)-(v) are global assumptions on operators $A, K$ and $B$ while (vi) is a local one depending on $u$ as a limit point of a sequence $\left(u_{n}\right)_{n \in \mathbb{N}}$.

- assumptions of Theorem 1 have been involved in those of Theorem 2 so that

$$
\forall u \in U \quad \mathcal{T}(u) \neq \emptyset .
$$

Proof.- We first prove (4.4).

With (2.4) and the convexity of $K\left(y_{n}\right)$ we claim that for any $t \in\left[0,1\left[\right.\right.$ then $t y_{n} \in K\left(y_{n}\right)$. As $y_{n} \in \mathcal{T}\left(u_{n}\right)$ we get

$$
\forall y \in K\left(y_{n}\right) \quad\left\langle A y_{n}-B u_{n}, y-y_{n}\right\rangle+\Phi\left(y, u_{n}\right)-\Phi\left(y_{n}, u_{n}\right) \geq 0,
$$

so that (with $y=t y_{n}$ )

$$
\forall n \in \mathbb{N}, \forall t \in[0,1] \quad(t-1)\left\langle A y_{n}-B u_{n}, y_{n}\right\rangle+\Phi\left(t y_{n}, u_{n}\right)-\Phi\left(y_{n}, u_{n}\right) \geq 0
$$

Now we use the first part of assumption (vi). Assuming (4.2), we obtain

$$
\left|\Phi\left(t y_{n}, u_{n}\right)-\Phi\left(y_{n}, u_{n}\right)\right| \leq L(1-t)\left\|y_{n}\right\|
$$

so

$$
\forall n \in \mathbb{N}, \forall t \in[0,1] \quad(t-1)\left\langle A y_{n}-B u_{n}, y_{n}\right\rangle+L(1-t)\left\|y_{n}\right\| \geq 0
$$

and

$$
\forall n \in \mathbb{N}, \quad\left\langle A y_{n}-A(0), y_{n}\right\rangle+\left\langle A(0)-B u_{n}, y_{n}\right\rangle-L\left\|y_{n}\right\| \leq 0 .
$$

We conclude with the strong monotonicity of $A$ that

$$
M\left\|y_{n}\right\|^{2} \leq\left(\|A(0)\|_{*}+L+\left\|B u_{n}\right\|\right)\left\|y_{n}\right\| .
$$

Alternatively, let us assume (4.2b); with (4.5) an $y=0$ we get

$$
\begin{gathered}
\forall n \in \mathbb{N}, \quad\left\langle A y_{n}-B u_{n},-y_{n}\right\rangle \geq \Phi\left(y_{n}, u_{n}\right)-\Phi\left(0, u_{n}\right) \geq 0, \\
\forall n \in \mathbb{N}, \quad\left\langle A y_{n}-B u_{n}, y_{n}\right\rangle \leq 0, \\
\forall n \in \mathbb{N}, \quad\left\langle A y_{n}-A(0), y_{n}\right\rangle+\left\langle A(0)-B u_{n}, y_{n}\right\rangle \leq 0,
\end{gathered}
$$

Once again the strong monotonicity of $A$ yields

$$
M\left\|y_{n}\right\|^{2} \leq\left(\|A(0)\|_{*}+\left\|B u_{n}\right\|\right)\left\|y_{n}\right\| .
$$

As the sequence $\left(u_{n}\right)$ is bounded and $B$ is compact we get (with (4.6) (respectively (4.7))

$$
\left\|y_{n}\right\| \leq \kappa(u)
$$


where $\kappa$ is a constant depending on $u$.

We now show point 2 : let $\left(y_{n}\right)_{n \in \mathbb{N}}$ be a sequence in $\mathcal{T}\left(u_{n}\right)$ such that $y_{n} \rightarrow y$ in $V$. We have to prove that $y \in \mathcal{T}(u)$ ).

First, observe that $y_{n} \in K\left(y_{n}\right)$ : so with (v), $y$ is an element of $K(y)$. Now, consider an arbitrary point $z$ in $K(y)$. Since $K(y)$ is convex, for all $t \in] 0,1], z_{t}:=t z+(1-t) y \in$ $K(y)$

We claim that

$$
\left\langle A\left(z_{t}\right)-B u, y-z_{t}\right\rangle+\Phi(y, u)-\Phi\left(z_{t}, u\right) \leq 0 .
$$

Indeed, according to (v) one can find a sequence $\left(z_{n}\right)_{n}$ (strongly) converging to $z_{t}$ such that $z_{n} \in K\left(y_{n}\right), \forall n \geq 1$. Since $y_{n} \in \mathcal{T}\left(u_{n}\right)$, for every $n \geq 1$, we have

$$
\left\langle A\left(y_{n}\right)-B u_{n}, z_{n}-y_{n}\right\rangle+\Phi\left(z_{n}, u_{n}\right)-\Phi\left(y_{n}, u_{n}\right) \geq 0 .
$$

Using the monotonicity of $A$ it follows that

$$
\left\langle A\left(z_{n}\right)-B u_{n}, z_{n}-y_{n}\right\rangle+\Phi\left(z_{n}, u_{n}\right)-\Phi\left(y_{n}, u_{n}\right) \geq 0 .
$$

The operator $B: U \rightarrow V^{\prime}$ is compact, so $\left(B u_{n}\right)_{n}$ strongly converging to $B u$ in $V^{\prime}$ ( up to a subsequence). Using hypothesis (iii) and (vi), up to subsequences if necessary, we obtain

$$
\begin{aligned}
\left\langle A\left(z_{t}\right)-B u, y-z_{t}\right\rangle+\Phi(y, u)-\Phi\left(z_{t}, u\right) \leq & \liminf _{n}\left\langle A\left(z_{n}\right)-B u_{n}, y_{n}-z_{n}\right\rangle \\
& +\liminf _{n} \Phi\left(y_{n}, u_{n}\right)-\underset{n}{\limsup } \Phi\left(z_{n}, u_{n}\right) \leq 0 .
\end{aligned}
$$

So, we have proved relation (4.8). We conclude that,

$$
\left\langle A\left(z_{t}\right)-B u, z_{t}-y\right\rangle+\Phi\left(z_{t}, u\right)-\Phi(y, u) \geq 0 .
$$

Using the convexity of $\Phi$ with respect to the first variable, we get

$$
\left\langle A\left(z_{t}\right)-B u, t(z-y)\right\rangle+t[\Phi(z, u)-\Phi(y, u)] \geq 0
$$

this gives

$$
\forall t \in] 0,1] \quad\left\langle A\left(z_{t}\right)-B u, z-y\right\rangle+\Phi(z, u)-\Phi(y, u) \geq 0
$$

We conclude with the hemicontinuity of $A$ that

$$
\langle A(y)-B u, z-y\rangle+\Phi(z, u)-\Phi(y, u) \geq 0
$$

As the inequality (4.10) holds for any $z \in K(y)$, we conclude that $y \in \mathcal{T}(u)$; this achieves the proof.

Example 1 Let us give a simple case where assumption (v) of Theorem 2 is satisfied . We define the set-valued mapping $K: V \rightrightarrows V$ by

$$
K(v)=K_{o}+m(v)
$$

where $K_{o}$ is a fixed closed convex and nonempty subset of $V$ and $m: V \rightarrow V$ is a compact map ( or equivalently weakly - strongly continuous ).

Proposition 1 For any sequence $\left(v_{n}\right)$ weakly convergent to $v$ in $V$, then $K\left(v_{n}\right)$ Moscoconverges to $K(v)$, where $K$ is given by (4.11). 
Proof.- Let $\left(v_{n}\right)$ be a sequence of $V$ such that $v_{n} \rightarrow v$, we have to prove that $K\left(v_{n}\right)$ Mosco-converges to $K(v)$. Let $w_{n} \in K\left(v_{n}\right)$ such that $w_{n} \rightarrow w$ in $V$. Since the operator $v \mapsto m(v)$ is compact $m\left(v_{n}\right) \rightarrow m(v)$. Since $K_{o}$ is weakly closed then, $w-m(v) \in K_{o}$. Hence, $w \in K(v)$.

Let $w \in K(v)$, then $\exists k \in K_{o}$ such that: $w=k+m(v)$. We set $w_{n}=k+m\left(v_{n}\right) \in K\left(v_{n}\right)$. It is clear that $w_{n} \rightarrow k+m(v)=w$. Therefore, $K\left(v_{n}\right)$ Mosco-converges to $K(v)$.

We will give in section 5 , some examples of such set-valued mapping $K$.

\section{The optimal control problem}

\subsection{Existence result}

Now we turn back to problem $(\mathcal{P})$ mentioned in Section 1 . We suppose that assumptions of Theorem 1 are satisfied so that the set-valued operator $\mathcal{T}$ (defined in the previous sections) is well defined on $U$.

Consider a cost functional $J: V \times U \rightarrow \mathbb{R} \cup\{+\infty\}$, we set

$$
(\mathcal{P}) \quad \min \left\{J(y, u), y \in \mathcal{T}(u), u \in U_{a d}, \subset U\right\},
$$

where $U_{a d}$ is a non-empty, convex and closed subset of the (Hilbert) space $U$.

Theorem 3 Assume $J$ is convex and lower-semicontinuous and either $U_{a d}$ is bounded or $J$ is coercive with respect to $u$. Assume assumptions of Theorem 2 are satisfied for every cluster point of minimizing sequences of problem $(\mathcal{P})$. Then problem $(\mathcal{P})$ has at least one optimal solution.

Proof.-The proof is straightforward. Let $\left(u_{n}\right)_{n \in \mathbb{N}} \in U_{a d}$ be a minimizing sequence. The boundedness of $U_{a d}$ or the coercivity of $J$ implies that $u_{n}$ is bounded in $U$. Let $u$ be a weak-cluster point of $\left(u_{n}\right)_{n \in \mathbb{N}}$ and denote the corresponding subsequence similarly. Assumptions of Theorem 2 are satisfied so that for every $y_{n} \in \mathcal{T}\left(u_{n}\right), y_{n}$ is a bounded sequence and weakly converges (up to a subsequence) to $y \in V$. Moreover $y \in \mathcal{T}(u)$. We end the proof with the lower semi-continuity of $J$ In the sequel, using [22] formalism we choose $J$ as follows :

$-\mathcal{N}: \mathcal{U} \rightarrow U$ is a linear, symmetric continuous and coercive operator i.e.

$$
\exists \kappa>0:\langle\mathcal{N} v, v\rangle \geq \kappa\|v\|^{2}, \forall v \in U
$$

- $\mathcal{H}$ is a Hilbert space of observations and $\mathcal{C} \in \mathcal{L}(V, \mathcal{H})$ be a given continuous operator.

- The desired state is $z_{d} \in \mathcal{H}$

We associate the following cost functional $J: V \times U \rightarrow \mathbb{R}$ defined by

$$
J(y, u)=\langle\mathcal{N} u, u\rangle_{U}+\left\|C y-z_{d}\right\|_{\mathcal{H}}^{2} .
$$

5.2 Some relevant examples

In this subsection, we will give some simple examples to support our theoretical results.

Example 2 Implicit Signorini problem 
Let $\Omega \subset \mathbb{R}^{n}$ an open bounded connected set with a regular boundary $\partial \Omega=\Gamma$. Let us consider the following implicit Signorini problem

$$
\left\{\begin{array}{l}
\text { Find } y \in K(y) \text { such that } \\
a(y, z-y) \geq\langle u, z-y\rangle, \quad \forall z \in K(y)
\end{array}\right.
$$

with the following data

$$
V=\left\{y \in H^{1}(\Omega) \mid \Delta y \in L^{2}(\Omega)\right\}
$$

for each $y \in V$, we associate the closed convex non empty set of $H^{1}(\Omega)$ defined by

$$
K(y)=\left\{z \in H^{1}(\Omega) \mid z_{\mid \Gamma} \geq h-\int_{\Gamma} \varphi \frac{\partial y}{\partial n} d \sigma \text { a.e. on } \Gamma\right\}
$$

with $h, \varphi \in H^{\frac{1}{2}}(\Gamma)$ and $h \geq 0$ on $\Gamma$.

$$
\begin{aligned}
& a(y, z)=\int_{\Omega}(\nabla y \cdot \nabla z+y z) d x \\
& \langle u, z\rangle=\int_{\Omega} u z d x, \quad u \in L^{2}(\Omega) .
\end{aligned}
$$

It is known that the solution $y$ of (5.2) (Implicit Signorini problem) is characterized by

$$
\left\{\begin{array}{l}
-\Delta y+y=u \text { a.e. in } \Omega \\
y \geq\left(h-\int_{\Gamma} \varphi \frac{\partial y}{\partial n} d \sigma\right), \quad \frac{\partial y}{\partial n} \geq 0, \quad\left[y-\left(h-\int_{\Gamma} \varphi \frac{\partial y}{\partial n} d \sigma\right)\right] \frac{\partial y}{\partial n}=0 \text { on } \Gamma
\end{array}\right.
$$

We have to verify assumptions of Theorem 2 :

- Assumption (i) is satisfied (we refer to [17] p.130) .

- It is clear that, (ii) and (iii) are ensured (with $A=-\Delta+I d$ ).

- $U=H=L^{2}(\Omega)$ and $B: H \rightarrow V^{\prime}$ is the canonical (compact) embedding, so we get (iv).

- Let us show that (v) is satisfied. We first note that $K$ is defined by (4.11) with

$$
K_{o}=\left\{z \in H^{1}(\Omega) \mid z_{\mid \Gamma} \geq h \text { a.e. on } \Gamma\right\}
$$

and $m: V \rightarrow V$ is given by $m(y)=-\int_{\Gamma} \varphi \frac{\partial y}{\partial n} d \sigma$, (here real numbers are identified to constant functions). We have to prove that $m$ is compact. Let $y_{k}$ be a sequence of $V$ weakly convergent to $y$. The normal derivative trace operator is linear, continuous (and thus weakly continuous) from $V$ to $H^{-\frac{1}{2}}(\Gamma)([23])$ so that $m\left(y_{k}\right) \rightarrow m(y)$ in $\mathbb{R}$. As the constant functions space (identified to $\mathbb{R}$ ) is (compactly) embedded in $V$ this gives the strong convergence of $m\left(y_{k}\right)$ to $m(y)$ in $V$.

$-\Phi=0$ so that (vi) is fulfilled.

We may summarize : 
Theorem 4 Assume $\alpha>0$. Then the optimal control problem

$$
\left\{\begin{array}{l}
\min \left\|y-z_{d}\right\|_{L^{2}(\Omega)}^{2}+\alpha\|u\|_{L^{2}(\Omega)}^{2} \\
-\Delta y+y=u \text { a.e. in } \Omega \\
y \geq\left(h-\int_{\Gamma} \varphi \frac{\partial y}{\partial n} d \sigma\right), \quad \frac{\partial y}{\partial n} \geq 0, \quad\left[y-\left(h-\int_{\Gamma} \varphi \frac{\partial y}{\partial n} d \sigma\right)\right] \frac{\partial y}{\partial n}=0 \text { on } \Gamma . \\
u \in U_{a d}
\end{array}\right.
$$

has (at least) one optimal solution.

\section{Example 3}

Let $\Omega \subset \mathbb{R}^{n}$ an open bounded connected set with a regular boundary $\partial \Omega=\Gamma$. We consider (5.2) with

$$
\begin{gathered}
V=H^{1}(\Omega) \\
a(y, z)=\int_{\Omega}\left[\sum_{i, j=1}^{n} a_{i j}(x) \frac{\partial y}{\partial x_{i}}(x) \frac{\partial z}{\partial x_{j}}(x)+b(x) y(x) z(x)\right] d x
\end{gathered}
$$

where the functions $x \mapsto a_{i j}(x)$ and $x \mapsto b(x)$ satisfy the following classical assumptions:

$$
a_{i j} \in L^{\infty}(\Omega), 1 \leq i, j \leq n, \quad b \in L^{\infty}(\Omega), b \geq 0 \text { a.e. on } \Omega
$$

and

$$
\begin{gathered}
\exists \beta>0, \forall \xi_{i}, 1 \leq i \leq n, \sum_{i, j=1}^{n} a_{i j} \xi_{i} \xi_{j} \geq \beta \sum_{i=1}^{n} \xi_{i}^{2} \text { a.e. on } \Omega . \\
K(y)=\left\{z \in H^{1}(\Omega) \mid \int_{\Omega}(z-y)(x) d x \geq 0\right\} .
\end{gathered}
$$

It is easy to check all assumptions of Theorem 2 except (v). Assume that $y_{k}$ is a sequence of $H^{1}(\Omega)$ weakly convergent to $y$. Let us prove that $K\left(y_{n}\right)$ Mosco-converges to $K(y)$ :

(i) Assume that $z_{n} \in K\left(y_{n}\right)$ weakly converges to $z$ in $H^{1}(\Omega)$. So $\left(z_{n}-y_{n}, 1\right)_{L^{2}(\Omega)} \geq 0$ and obviously converges to $(z-y, 1)_{L^{2}(\Omega)}$. Therefore $\int_{\Omega}(z-y)(x) d x \geq 0$ that is $z \in$ $K(y)$.

(ii) Let $z \in K(y)$. We set $z_{n}=z+\int_{\Omega}\left(y_{n}-y\right)(x) d x$. Obviously $z_{n}$ strongly converges to $z$ in $H^{1}(\Omega)$. Moreover

$$
\int_{\Omega}\left(z_{n}-y_{n}\right)(x) d x=\left(z_{n}-y_{n}, 1\right)_{L^{2}(\Omega)}
$$

$=\left(z+\left(y_{n}-y, 1\right)_{L^{2}(\Omega)}-y_{n}, 1\right)_{L^{2}(\Omega)}=\left(z-y_{n}, 1\right)_{L^{2}(\Omega)}+\left(y_{n}-y, 1\right)_{L^{2}(\Omega)}=(z-y, 1)_{L^{2}(\Omega)} \geq 0$,

that is $z_{n} \in K\left(y_{n}\right)$.

Now it is easy to conclude as previously.

Example 4 Implicit Obstacle problem with friction 
Let $\Omega \subset \mathbb{R}^{2}$ be the section of a tube with infinity length $\left.\Omega \times\right]-\infty,+\infty[$ and a thin membrane spanned in the tube held by a thin elastic wire. We assume that $\Omega$ is an open bounded connected set with a regular boundary $\partial \Omega$. The membrane deforms under the action of a surface density force $f$ acting in the axe-direction and is required to stay on or above an obstacle $m(u)$ where $m: H^{1}(\Omega) \rightarrow H^{1}(\Omega)$ is a compact operator. The forces $f$ are balanced by the force that the obstacle exerts on when the membrane is in contact with it. The displacement of the membrane is governed by the quasi-variational inequality (5.2) where

$$
\begin{gathered}
V=H^{1}(\Omega), K(y)=\{z \in V \mid z \geq m(y)\}, a(y, z)=\int_{\Omega}(\nabla y \cdot \nabla z+y z) d x \\
\langle f, y\rangle=\int_{\Omega} f y d x \text { and } \Phi(y)=\int_{\Gamma} g|y| d \sigma
\end{gathered}
$$

with $g \in L^{\infty}(\Gamma), g \geq 0$ on $\Gamma$ a given friction bound.

Assumptions of Theorem 2 are satisfied. The function $\Phi$ is convex and Lipschitz continuous and it is easy to see that

$$
K(y)=K_{o}+m(y)
$$

as in (4.11) with

$$
K_{o}=\left\{z \in H^{1}(\Omega) \mid z \geq 0 \text { a. e. in } \Omega\right\},
$$

So we conclude that Theorem 2 applies.

\section{Conclusion}

We have given an abstract framework that allows to consider optimal control problems governed by Quasi-Variational Inequalities. The strong monotonicity of the operator $A$ and the Mosco-convergence of the set-valued application $K$ involved in the QVI are key assumptions in the proofs presented in this paper. Since in some applications the operator $A$ is only semi-coercive and since the underlying compactness assumption (involved in the Mosco-convergence of $K$ ) does not allow to deal with "usual " constraints as pointwise constraints for example. It would be be very interesting to obtain similar results with only a monotonicity assumption insteact of a compactness one. This is out of the scope of the present manuscript and will be the subject of another work.

\section{References}

1. S. Adly, M. Ait Mansour and L. Scrimali, Sensitivity analysis of solutions to a class of quasi-variational inequalities, Bollettino U.M.I (8) 8-B (2005), 767-771.

2. Aubin, J.-P. And Frankowska, H., Set-valued analysis, Birkauser, 1990.

3. Aussel F. AND LuC D.T, Existence conditions in general quasimonotone variational inequalities, Bull. Austral. Math. Soc. 71, 2,pp.285-303, 2005,

4. C. Baiocchi AND A. CAPelo, Variational and Quasi-variational Inequalities. Wiley: New York, 1984.

5. V. BARBU, Analysis and Control of Non Linear Infinite Dimensional Systems, Math. Sci. Eng.. 190, Academic Press, San Diego, 1993.

6. A. Bensoussan And J.L Lions, Contrle impulsionnel et inéquations quasi-variationnelles, Dunod, Paris (1982). 
7. M. Bergounioux and S. Lenhart, Optimal control of bilateral obstacle, Siam Journal On Control and Optimization, Vol 43, n1, pp. 240-255, 2004

8. J. Borwein AND Q. J. ZHu, Techniques of variational analysis, An introduction, Springer, 2004.

9. H. Dietrich , A Smooth Dual Gap Function to a Class of Quasivariational Inequalities, JMAA, Vol. 235, pp. 380-393, 1999.

10. H. Dietrich , Optimal control problems for certain quasivariational inequalities, Optimization, Vol. 49, pp. $67-93,2001$.

11. A. Dontchev And T. ZolezzI, Well posed optimization problems, Lecture notes in mathematics, 1543, Springer (1993).

12. G. Duvaut And J.L. Lions, Les inéquations en Mécanique et en physique, Dunod, 1972, Inequalities Mechanics and Physics, Berlin, Heidelberg, New York, 1976.

13. F. FloRES BAZÁn, Existence theorems for generalized noncoercive equilibrium problems: The quasi-convex case, SIAM J.Optimization , Vol. 3, pp. 675-690, 2000.

14. F. Gianessi And A. MAugeri, Variational Inequalities and Network Equilibrium Problems Applications, Plenum Press, New-York, 1995.

15. A. Granas And J. Dugundu, Fixed point theory. Springer Monographs in Mathematics. Springer-Verlag, New York, 2003.

16. M. Hukuhara, Sur lexistence des points invariants dune transformation dans lespace fonctionnel, Japan. J. Math. 20 (1950) 14

17. J-L. Joly And U. Mosco, A propos de l'existence et de la régularité des solutions de certaines inéquations quasi-variationnelles, J. Func. Anal. , 34, 107-137, 1979

18. D. Kinderlehrer And G. Stampacchia, An introduction to Variational Inequalities and Their Applications. Academic Press, New York, 1980

19. M. Kunze AND J.F. Rodrigues, An elliptic quasi-variational inequality with gradient constraints and some of its applications, Math. meth. Appl. Sci 897-908, 2000

20. M. B. Lignola, Well posedness and L-wellposedness for quasivariational inequalities, Journal of Optimization Theory and Applications, Vol. 128, 1, pp 119-138, 2006.

21. M. B. Lignola And J. Morgan, Convergence of solutions of Quasi-Variational Inequalities and Applications, Topological Methods in Nonlinear Analysis, Vol. 10., 1997

22. J.L Lions, Contrle optimal de systmes gouvernés par des équations aux dérivées partielles, Gauthier-Villars, Paris (1968).

23. J.L Lions And E. Magenes, Problmes aux limites non homognes et applications, Vol. 1, Dunod, Paris (1968).

24. P.L. Lions, Two remarks on the convergence of convex functions and monotone operators, Nonlinear Analysis 2, pp 553-562 (1978).

25. U. Mosco, Convergence of convex sets and of solutions of variational inequalities, Adv. in Math. 3, 510-585, 1969

26. Y. Sonntag, Convergence au sens de U. Mosco : théorie et application à l'approximation des solutions d'inéquations, Thèse, Université de Provence, 1982

27. E. ZEIDLER, Nonlinear Functional Analysis and Its Applications , Springer, Berlin 1992. 